DOI: $10.2478 / v 10122-010-0016-8$

\title{
LEXICA AFROASIATICA IX
}

\author{
GÁBOR TAKÁCS
}

\begin{abstract}
Gábor Takács. Lexica Afroasiatica IX. Lingua Posnaniensis, vol. LII (2)/2010. The Poznań Society for the Advancement of the Arts and Sciences. PL ISSN 0079-4740, ISBN 978-83-7654-078-8, pp. 85-98
\end{abstract}

Comparative-historical Afro-Asiatic linguistics has undergone significant development over the past half century, since the appearance of Essai comparatif sur le vocabulaire et la phonétique du chamitosémitique (1947) by Marcel CoHEN. This revolutionary and fundamental synthesis concluded the second great period of the comparative research on Afro-Asiatic lexicon (the so-called "old school", cf. EDE I $2-4)$. During the third period (second half of the $20^{\text {th }}$ century), whose beginning was hallmarked by the names of J.H. Greenberg and I.M. Diakonoff, a huge quantity of new lexical material (both descriptive and comparative) has been published, including a few most recent attempts (either unfinished or rather problematic) at compiling an Afro-Asiatic comparative dictionary (SISAJa I-III, HCVA I-V, HSED, EHRET 1995).

During my current work on the Etymological Dictionary of Egyptian (EDE), I have collected a great number of new AA parallels, which - to the best of my knowledge - have not yet been proposed in the literature or were observed independently from me. ${ }^{1}$ Along the EDE project (and the underlying "Egyptian etymological word catalogue"), I have started collecting AA roots (not attested in Egyptian) for a separate Afro-Asiatic root catalogue in late 1999.

The series Lexica Afroasiatica started in $2002^{2}$ for communicating new Afro-Asiatic lexical correspondences observed recently during my work, which may later serve as basis of a new synthesis of the Afro-Asiatic comparative lexicon. The present part of this series is a collection of additional new AfroAsiatic etymologies with the Proto-Afro-Asiatic initial bilabial nasal $\left({ }^{*} m-\right)$ observed after my research periods at Institut für Afrikanische Sprachwisenschaften of Frankfurt a/M (in 1999-2000 and 2002) guided by Prof. H. Jungraithmayr. The numeration of the etymological entries is continuous beginning from the first part of the series Lexica Afroasiatica.

Each entry is headed by the proposed PAA root (as tentatively reconstructed by myself). Author names

1 I did my best to note it wherever I noticed an overlapping with the existing Afro-Asiatic dictionaries.

2 The first part of this series (lexical parallels with with PAA *b-) appeared in Afrikanistische Arbeitspapiere (Köln) 67 (2002), 103-151. The second part (additional lexical roots with AA *b-) was published Kogan, L. (ed.): Orientalia: Papers of the Oriental Institute, Issue III: Studia Semitica (Moscow 2003, Russian State University for the Humanities, pp. 331-348). My paper "Lexica Afroasiatica III" (new AA roots with *p-) appeared in Takács, G.: Egyptian and Semito-Hamitic (Afro-Asiatic) Studies in Memoriam Werner Vycichl (Leiden 2003, E. J. Brill, pp. 510-550), while "Lexica Afroasiatica IV" (with new etymologies with AA * $f$-) was published in Acta Orientalia Academiae Scientiarum Hungariae (Budapest) 57/4 (2004), 457-473. "Lexica Afroasiatica V" (new AA roots with * $\dot{p}$-, ${ }^{*} P$-) appeared in Studia Etymologica Cracoviensia 9 (2004), 159-178. The sixth issue (roots with * $m$ - followed by dentals, palatals, laterals, velars, pharyngeals, and laryngeals) is forthcoming in the series of GLECS (Comptes Rendus du Groupe Linguistique d'Études Chamito-Sémitiques). The seventh part (roots with *m- followed by sonorants) was published in Takács, G. (ed.): Semito-Hamitic Festschrift for A. Dolgopolsky and H. Jungraithmayr. Berlin 2008, Dietrich Reimer Verlag, pp. 310-336. "Lexica Afroasiatica VIII" (containing additional roots with * $m$-) appeared in Rocznik Orientalistyczny (Warszawa) 62/2 (2009), 26-125. 
are placed after the quoted linguistic forms in square brackets [] mostly in an abbreviated form (a key can be found at the end of the paper). The lexical data in the individual lexicon entries have been arranged in the order of the current classification of the Afro-Asiatic daughter languages (originating from J. H. Greenberg 1955; 1963 and I. M. Diakonoff 1965) in 5 (or 6) equivalent branches: (1) Semitic, (2) Egyptian, (3) Berber, (4) Cushitic, (5) Omotic (cometimes conceived as West Cushitic), (6) Chadic. For a detailed list of all daughter languages cf. EDE I 9-34. The number of vertical strokes indicates the closeness of the language units from which data are quoted: ||| separate branches (the 6 largest units within the family), || groups (such as East vs. South Cushitic or West vs. East Chadic), while | divides data from diverse sub-groups (e.g., Angas-Sura vs. North Bauchi within West Chadic).

Since we know little about the Proto-Afro-Asiatic vowel system, the proposed list of the reconstructed Proto-Afro-Asiatic forms is arranged according to consonantal roots (even the nominal roots). Sometimes, nevertheless, it was possible to establish the root vowel, which is given in the paper additionally in brackets. The lexical parallels suggested herein, are based on the preliminary results in reconstructing the consonant correspondences achieved by the Russian team of I. M. Diakonoff (available in a number of publications ${ }^{3}$ ) as well as on my own observations refining the Russian results (most importantly TAKÁCS 2001). The most important results can be summarized as follows. The labial triad $* b-{ }^{*} p-{ }^{*} f$ remained unchanged in Egyptian, South Cushitic, and Chadic, while the dental series * $d-* t-t$ was kept as such by Semitic and South Cushitic (AA * $t$ continued as * $d$ in Berber, Cushitic and Chadic, and it was merged into $t$ vs. $d$ in Egyptian). The fine distinction of the diverse sibilant affricates and spirants

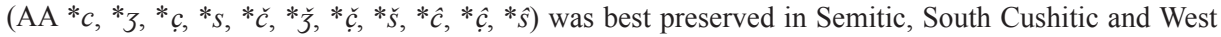
Chadic (while some of these phonemes suffered a merger in other branches and groups). The Russian scholars assumed a triad of postvelar (uvulear) stops with a voiceless spirant counterpart: ${ }^{*} g$, ${ }^{*} \dot{q}$, $* q$, and $* h$, the distinction of which was retained in Cushitic and Chadic, but was merged into $* h$ in Semitic and Egyptian. In a number of cases, however, it is still difficult to reconstruct exactly the root consonants on the basis of the available cognates (esp. when these are from the modern branches, e.g., Berber, Cushitic-Omotic, or Chadic). In such cases, the corresponding capitals are used (denoting only the place of articulation). ${ }^{4}$

This part contains new Afro-Asiatic roots with $* n$ - followed by a labial.

Gábor Takács, Székesfehérvár 8000, Semmelweis u. 19, Hungary, gabtak@mail.datatrans.hu

\section{$\mathbf{A A} * n-$}

866. AA * $\boldsymbol{n}$ - $\boldsymbol{b}$ "odor, smell" > Sem.: Ar. nabb-at- "odeur désagréable, fétide" [BK II 1178] ||| ECh.: WDangla níbé \& EDangla nibē "sentir (avec le nez), flairer” [DM 1973, 218] = EDangla nibe "riechen” [Ebs. 1979, 126; 1987, 91], Migama núbáw “sentir (odeur), flairer, puer" [JA 1992, 110], Bidiya nuub "sentir, flairer" [AJ 1989, 102]. This Ar.-ECh. etymology was observed independently also by Stolbova $(2005,111, \# 387)$.

867. AA * $\boldsymbol{n}$ - $\boldsymbol{b}$ "to call by name" > Sem. * $n \boldsymbol{b}$ ? (tr.) "to name, proclaim" [Hnrg. 1999, 91, \#3] $>$ e.g. Akk. nabû G "1. (mit Namen) nennen, 2. jmdn. berufen, 3. verordnen" [AHW 699] III SOm. (Aroid) *nab- *nam- "name" [Bnd. 2003] = *nāb- (from **na?b-?) "name" [GT]: Ari nāmi [Bnd. 1971, 263, \#55] = nām- [Bnd. 1994, 155] = nam-i [Bnd. 1994, 1159, \#55], Banna nābi [Bnd. 1971, 264, \#55], Galila lām- [Bnd.], Hamer nam- nab- [Lydall apud Bnd.], Dime napp-o [Bnd. 1971, 263, \#55] = nāp- [Flm./Bnd. 1994] $=n \bar{a} b-i / o$ [Flm./Bnd. 1996 MS, 2, \#55] = nāf-u [Bnd. 1996 and 2003] (SOm.: Bnd. 1994, 155; 2003, 214, \#92). The Sem.-SOm. etymology was recorded independently by EHrET (1995, 315, \#610).

3 Cf. Diakonoff 1984; 1988: 34-41; 1992; Diakonoff et al. 1987; 1993; SISAJa I-III; HCVA I-V.

4 E.g., the symbol (*P-) signifies any unknown initial labial, just as, e.g., *T stands for any unknown dental stop $(* d, * t$ or $* t)$ or $* K$ for any unknown velar stop $(* g, * k$ or $* k)$ or $* Q$ for any unknown postvelar/uvulear $(* g, * q$ or $* \dot{q})$, while $* H$ for any laryngeal or pharyngeal $(* ?, * h, * r, * h$, also $* \gamma)$. 
868. AA * $\boldsymbol{n}-\boldsymbol{b}$ "1. big, 2. old" > Sem. *nyb *nwb "to be big, elder" [GT]: Ar. $n y b: n \bar{a} b a$ II "3. être très-âgé (se dit surtout d'une chamelle)", nāb-, pl. nuyūb- ?anyub- ?anyāb"1. dent canine, 2. (de là) chef d'une tribu, d'une communauté, le premier parmi les siens" [BK II 1375] = nāb- "alte Kamelin" [Rdk.] = nāb-, pl. ?anyāb- "1. (f) dent canine, litt. la 'grande' dent, 2. (m) chef de tribu, d'une communauté et son gran homme" [Vcl. in DELC pace Lisān $a l-\Upsilon_{a r a b}=n \bar{a} b$ - "1. she-camel (sic), 2. head of family" [Lsl. 1962, 67] = nāb"1. Stammeshäuptling, 2. alte Kamelstute" [Vcl. 1960], cf. also Ar. nawb- "power" [Vollers, ZA 23, 1909, 105], Yemeni Ar. $n w b: n \bar{a} b$ (impf. yinūb) "to rule" [PiAmenta 1990, 499] ॥ MSA * $n w b$ : Hrs. e-nōb, nyōb "big" [Jns. 1977, 99], Mehri $a-n \bar{b} b$ (f) "groß, älter" [Rdk. 1911, 22, §110] = nōb, pl. nəyōb "big" [Jns. 1987, 306] = ha-nōb "grand, âgé" [LSS 1983, 195], Mehri of Qishn $a-n \bar{b} b$ (f) "big (grande)" [SS, also LonNeT 1993, 66, fn. 38], Bathari nawb "big" [Morris/SS] (MSA: SS 1997, 393) || ES: (?) Geez nop nāp [irreg. - $p$ ] "deacon, Our Lady, strong” [Lsl. 1987, 398] (Sem.: Lsl. 1962, 67) ||| Eg. nb "Herr" (OK-, Wb II 227-230) ||| LECu.: Saho nab "groß sein, werden, erwachsen", nab "groß, mächtig, vornehm", ma-nab-ōytā "der von der Größe" [Rn. 1890, 281-282] = nab-a "to be big, great", nab-inta nabne "greatness, bigness, growth", ma-nab-otta "leader of the chiefs, president, governor" [VERGARI 2003, 131, 138-139], Saho-Assaorta nab "essere grande, crescere” [CR 1913, 72], Afar nab "groß sein, werden", ma-nab-ōytā "der Chef, Anführer" [Rn. 1886, 887-888] = nab "essere grande, crescere" [CR 1913, 72] = nab-a "to be big, great, many" [PH 1985, 173], Rendille náb-o "Versammlungsplatz der Senioren im Zentrum einer jeden Siedlung" [SCHLEE 1978, 142, \#804] = násb-o "fenced circle of the elders (in the middle of a village), elders' meeting-place (within the clan-village) where elders meet every night to chat and make decisions about matters affecting the village" [PG 1999, 230], Arbore níb "greatly, very" [Hyw. 1984, 388] | HECu.: Kambatta nub-áčču "old", nubáṕpe? "to be old"

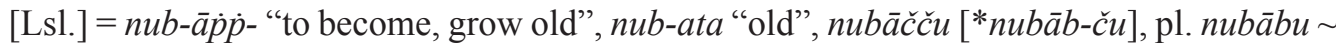

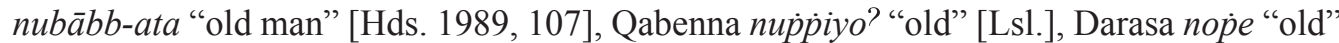
[Lsl.] (HECu.: Lsl. 1956, 988). For Eg.-LECu.: Rn. 1886, 887-888. For Eg.-Ar.: EMBER 1917, 83, \#101; DELC 138; Rsl. 1971, 310; Vernus 2000, 189. For Eg.-Mhr.-Ar.-LECu.: Müller 1975, 68, \#58. For the Eg.-Ar.-MSA etymology see Vcl. 1960, 174-5, \#4.

869. AA * $\boldsymbol{n}$ - $\boldsymbol{b}$ "to rise" > Sem.: Akk. $n a b \bar{a}^{?} u$ "(etwa) aufsteigen, aufsprudeln (?)" [AHW 697] = "to rise (said of flood)" [CAD n1, 24] || OSA (Sabaean) $n b$ " "level, terrace of cultivated land" [Biella 1982, 290], Ar. naba?a "1. être haut, élevé, 2. paraître au dessus de la tête de qqn., aparraître dans un endroit plus élevé que nous sommes" [BK II 1178] = "hoch sein, von oben kommen" [AHW] (for Sem. biconsonantal *nb- see JuŠMANOv 1998, 133) ||| (?) Eg. $n b n b$ (unless < bnbn) "vom Kommen der Überschwemmung" (GR, Wb II 245, 7) = "to bring forth" (PL 505) ||| ECu.: Tsamay nebeh- "to exceed, surpass" [Hyw. 1989, 13].

870. AA * $\boldsymbol{n}$ - $\boldsymbol{b}$ "hollow" > Sem. * $n b b$ : Ug. * $n b b$ "to empty out (Aqht's remains from the fem. eagle)" [Gordon 1955, 293, \#1193 pace Ullendorff, Or. 20, 1951, 272-273] = "to hollow out, eviscerate" [Gordon apud KB] = "aushöhlen, ausweiden" [WUS \#1734] = "to hollow (?)" [Segert], Hbr. *nbb "hohl sein, aushöhlen", qal part. pass. nābūb "1. hohl, 2. (übertragen) leerköpfig, dumm" [GB 479] = *nbb "to be hollow" [KB 659], MHbr. nābūb "hohl" [DALMAN 1922, 260] $=$ "1. hollow, 2. hungry" [JASTROw 1950, 867], Samar. Aram. nbb "to be hollow" [TAL 2000, 494] (Ug.-Hbr.: DAhood 1976, 360; SEgerT 1984, 193) ||| Eg. nb.t "voûte, encourbellement" (NK, AL 77.2048) = "Gewölbe, Laube" (GHWb 401) = "tonnelle 
(désigne certainement une petite construction ombragée destinée au réjouissances; il est tentant d'identifier cette construction avec le kiosque figuré parfois dans les jardins décorant les tombes du Nouvel Empire, et qui pouvait servir à conserver les boissons fraîches" (MA-

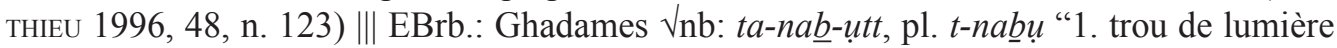
rectangulaire aménagé au centre du plafond pour éclairer la pièce principale d'habitation" [Lanfry 1973, 232, \#1076] || LECu.: Saho \& Afar nabe ${ }^{\Upsilon} \bar{e}$, pl. ná́be $(f)$ [-` body parts' suffix in ECu.] "Achselhöhle" [Rn. 1886, 888; 1887, 280; 1890, 283].

NB: Mathieu (1.c.) assumed a connection with Eg. nb "basket", "dont le nom proviendrait peut-être, par métonymie, de son toit en forme de corbeille renversée".

871. AA * $\boldsymbol{n}-\boldsymbol{b}$ "to care for" > Sem.: Ar. nabnaba "3. rester longtemps à faire qqch. pour en soigner le travail”" [BK II 1189] ||| Eg. nbj.w "der Schützer jemds., einer Sache” (NK, Wb II 245, 1-2), nbnb "bewahren, (be)schützen, in Ordnung halten" (XVIII., Wb II 245, 4-6) = "to protect" (PL 505) ||| ECh.: Bidiya nebew "garder les enfants" [AJ 1989, 101].

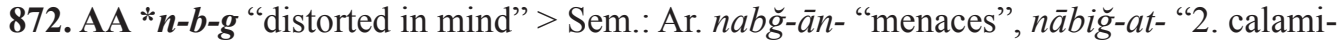
té, malheur" [BK II 1182] ||| Eg. nb $\underline{d}$ [reg. < *nbg] "böse, schädlich" (OK-, Wb II 247, 4) = "schlecht, übel, schädlich, zerstörerisch sein" (GHWb 406) ||| Brb. *n- $\underline{b}-g^{y}[\mathrm{GT}]>$ Ahaggar a-nhag \& Ghat a-nhağ "être sans bon sens" [Prs. 1969, 82, \#542] = Ghat e-nhež "être fou" [Nhl. 1909, 162], Ayr a-nnə-nbəg "1. chute, 2. obsession érotique, sexuelle" [PAM 2003, $582]$.

873. AA *n-b-r "spindle" > Eg. nb3 [reg. < *nbr] "Spindel" (CT III 133b, KHW 87, 519; NBÄ 783-6, n. 980; Osing in LÄ III 948; OsING 1980, 140) = "fuseau” (AL 77.2065, 78.2062)

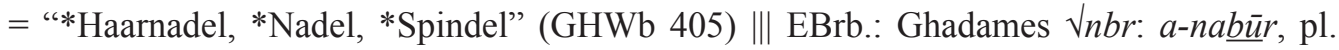
ne $\underline{b} r-\bar{a} n$ "ensouple du métier à tisser" [LANFRY 1973, 233, \#1082].

874. AA * $\boldsymbol{n}$ - $\boldsymbol{b}$ - $\boldsymbol{l}$ "vessel for liquid matter" > Sem.: Ug. $n b l$ "flagon" [GoRdon 1955, 294, \#1199] = "jar, amphoras, skin-bottle, pitcher" [DUL 618 pace Pardee contra DL in UF 17, 1986, 143], Hbr. nēbel nebel "1. Schlauch, Weinschlauch, 2. Krug, Geschirr, Gefäß” [GB 481] = "skin-bottle (not pitcher)" [GuILlaUME 1965 II, 25] = "(usually rendered jar, bottle, wineskin, flagon as term of measurement; no doubt, it refers to a vessel or container used for liquids; occasionally, it refers to the volume of the contents of a designated vessel)" [JACOBSON 2001, 84], Punic $n b l$ "jar (?)" [HARRis 1936, 123] = “сосуд” [ŠIFMAN 1963, 64] = "(indicating certain type of vessel containing wine, oil, of cast metal etc.)" [DNWSI 712], MHbr. nēbel nebel "Lederflasche" [DALMAN 1922, 261] = "1. leather bottle, 2. a hollow musical instrument with a leather body" [JASTROw 1950, 869] ||| Eg. nb3 [reg. <*nbl] "ein Gegenstand" (CT, Wb II 243, 11) = "ointment-horn” (AECT I 165, spell 203, n. 10) = "ointment-horn, censer, some object from which a liquid is poured or sprinkled (its strange determinative is seen in the objects used for sprinkling water in rituals)" (WARD 1978, 21, \#29 \& 110, \#216 \& 111, \#217; cf. Osing 1980, 139: "etymologisch bisher nicht durchsichtig, Einordnung unsicher”) = "*Horn (mit Salbe gefüllt)" (GHWb 405).

875. AA *n-p "to spray water" > Sem. *np "to besprinkle" [Zbr.]: Ug. $n p p$ Gt "to sprinkle o'self, anoint o'self” [DUL 635], Hbr. nwp I qal "besprengen" [GB 493] = "to spray with perfume" [KB 682] | Ar. naffa "to sprinkle", ?anfäf- "drizzle" [KB] vs. nafnafa "bruiner", nafnăf- "bruine, petite pluie froide très-fine" [Dozy II 706] = nafnafa "to mizzle, drizzle" 
[Zbr.] vs. $n f w / y$ : nafā "verser, répandre (se dit d'un nuage qui verse l'eau)", nafiyy- "2. un peu d'eau qui tombe de la corde du seau sur le dos de celui qui tire de l'eau, 3. qques gouttes de pluie" [BK II 1317], Dathina $n f f$ "plevoir fin, bruiner", nafäf- nafnāf- "pluie fine" [GD 2804, 2810], Yemeni Ar. naff "to drizzle" [Piamenta 1990, 491] || Geez näfnäfä "to besprinkle" [Lsl.] = nafnafa "to besprinkle, bedew" [Zbr.] = nafnafa "to drizzle, fall in small drops", nəfnəf nə/afnif "drizzle, shower, moisture, dro/ip" [Lsl.], Tigre nafnafa "to spray" [LH 347b], Tna. nifnif bälä "a light rain falls" [Lsl.] (Sem.: Lsl. 1958, 33; 1987, 388; Zbr. 1971, \#157) || Eg. npn “das Wasser” (GR, Wb II 248, 10) ||| LECu.: Afar nắfu (m) "dew” [PH 1985, 172] ||| CCh.: Margi nànafo "sprinkled" [LAdEFoged 1964, 65] | Mada ánaf "rosée, sueur" [Brt.-Bléis 2000, 205].

876. AA * $\boldsymbol{n}$ - $\boldsymbol{p}$ "seed, grain" > ES: Geez nappa "to produce fruit", nāpnāp "fruit", nopnop "bunch of fruit" [Lsl. 1987, 398] ||| Eg. npn.t "Getreidekorn" (PT, Wb II 248, 13; GHWb 406) = "Korn, Getreide" (Osing in NBÄ) > Cpt. (S) Na $\mathbf{n} €$, (M) N€ாN€ (f) "Korn" (KHW 124), usually regarded as sub-form (Nebenform) of Eg. $n p r \sim n p j>$ (S) Na/€ TP€ (cf. Fеснт 1960, 9, fn. 26; Hornung 1963 II, 55, n. 187; CED 110; NBÄ 749, n. 910 with a different etymology; KHW 124; DELC 144) ||| SBrb.: ETawllemmet \& Ayr ta-nufay-ăt "esp. de céréale (ou la graine de celle-ci?)” [PAM 2003, 597].

877. AA * $\boldsymbol{n}$ - $\boldsymbol{p}-\boldsymbol{k}$ "well" > Sem.: Ug. $n p k \sim n b \boldsymbol{k}$ "well" [GoRdON 1955, 298, \#1263 and 294, $\# 1198$ ] = npk $n b k$ "Quelle" [WUS \#1819, \#1738] = npk nbk "fountain, spring" [DUL $617]$ = syll. NABKU "well, spring (in field designation)" [Hnrg. 1987, 151], Hbr. *nēbek, in: nibkē-yām "Quellen des Meeres" [GB 480] = "sources of water" [KB 663] ||| Eg. npt (old *npt reg. <*npk) in: npt-jtr.w "als Stelle, von der Wasser entnommen wird” (GR, Wb II $249,19)$.

878. AA * $\boldsymbol{n}-\boldsymbol{p}-\boldsymbol{h}$ "lower part of belly" > Sem.: Ar. ?infah $(h)$-at- $\sim$ ?infih-at- $\sim m /$ binfah-at"the rennet or rennet-bag, of a kid or lamb [Lane 2821] = 'infah-at- $\sim$ ?infihh-at- "ventricule d'un agneau ou d'un chevreau" [BK II 1306] ||| Eg. nph.(w) "Teil des menschlichen Unterleibes (in der Gegend der Genitalien): ob Leistengegend?" (Med., Wb II 249) = "Hüften, Lenden" $($ Stern) = "pis" (Montet) = "les aînes, inguina" (Loret 1896, 176-179, §1) = "vermutlich: Kreuzbeingegend" (EBBELL 1937, 305-307) = "vorn unten am Leibe zu lokalisieren: Leistengegend, Euter der Kuh" (GRAPOw 1954, 83-84) = "iliac region" (FD 130) = "Beckenraum, Leistengegend, Unterleibsregion, Euter" (Westendorf 1966, 143) = "Beckenraum" (GHWb 407) = "rear parts, udder" (Smith 1.c.) = "inguinal lymph nodes, region, groin" (WALKER 1996, 251-256) = "groin" (PL 512). For further parallels cf. TAKÁCs 2004, 59, \#349.4.

879. AA *n-p-r "entrails" > Eg. np3 [reg. < *npr *npl] "Darm" (CT, Wb II 247, 12) = "navel-string" (CT IV 149, AECT I 251, spell 322, n. 4) = "1. (CT IV 149a) intestine, 2. (CT VI 441) navel-string, umbilical cord" (Walker 1996, 270) = "navel-string, entrails" (DCT 220) ||| NBrb.: Senhazha $\sqrt{n f r}$ : e-nneffar "gros intestin" [Rns. 1932, 392] | Qabyle $\sqrt{n f r}$ : ti$n e f f e r-t$ "panse des ruminants" [Dlt. 1982, 552]. A false LECu.-WCh. etymology of Eg. np3 (AA *l-p "spleen" [GT]) was suggested in OS 1992, 171; 1992, 186 and rejected by TAKÁCS 2004, 206, \#914.

880. AA *n-p-r "rim (of a well)" > Sem.: Akk. nap/burru "Zinne" [AHW 700] = naburru 
"battlements" [CAD n, 40] || Jbl. núfur "cut edge of a pit and the soil piled there (which might cause one to lose one's footing)" [Jns. 1981, 182] ||| Eg. npr.t "Rand eines Beckens und Brunnens, Ufer des Ozeans und des Nils" (MK-, Wb II 249, 8-10) ||| EBrb.: Ghadames a-nīfer, pl. nifar-āw-en "pan de bournous ou de houli" [LANFRY 1973, 237, \#1111] ॥ SBrb.: Ahaggar $a$-fer, pl. i-ferr-en "pan (d'un vêtement qui a 2 extrémités pendantes)" [Fcd. 1951-1952, 336-337].

881. AA * $\boldsymbol{n}$-f "fresh" > Eg. $n f$ "vom Bier: Erfrischung (?)" (GR, Wb II 250, 9) ||| SBrb.: ETawllemmet \& Ayr $\breve{a}$-ṇaf a- "pâturage frais, bons pâturages", ETawllemmet a-năfo "vent frais et humide provenant de la pluie" [PAM 2003, 592-593] || LECu.: Rendille nếf "coolness", néfowa "to become cool, cool down (mostly of liquids and foods)" [PG 1999, 233].

882. AA * $\boldsymbol{n}-\boldsymbol{f}$ "to chase, abandon, avoid" > Sem. *npp: MHbr. npp piel "treiben lassen" [Dalman 1922, 274] | Dathina $n f f$ "repousser brusquement, donner un coup transversal" [GD 2804] || NBrb.: Shilh a-nef "fermer, (s')écarter, s'abriter, changer de direction" [JoRDAN 1934, 35], Tazerwalt $\ddot{a}-n f$ "sich seitwärts wenden, abbiegen, beiseiterücken" [STUMME 1899, 165], Sus $a-n f$ "changer la direction, se mettre à l'écart" [Lst. 1921, 286] | Zayan a-nef "s'écarter" [Lbg. 1924, 575], cf. Tamazight $\sqrt{n f}$ : ssenfu "éviter (d'affronter qqn. par peur), ne pas répondre aux provocations, ignorer qqn." [TAIFI 1991, 469] | Qabyle a-nef 1. s'écarter du chemin, 2. laisser" [Dlt. 1982, 547], Zwawa \& Bugi a-nef "laisser, abandonner" [Bst. 1890, 324; Blf. 1910, 208] ||| WCh.: PGoemay *nēp (?) "to yield, give way to" [TAKÁCS 2004, 267]: Goemay nèp, pl. nap "to deviate, step aside" [Srl. 1937, 157] = neep "to give way" [Hlw. 2000 MS, 25], cf. also Montol puss-neep "afternoon", puus-neep “evening” (lit. „[when] sun yields [to night]”?, AS *pūs “sun”) [Ftp. 1911, 214, 216].

883. AA * $n-f$ "to rise, swell" > Sem. *nwp (*nūp) "hoch sein" [DL]: Ug. $n p$ "height" [Caquot/KB] $=n p(\mathrm{~m})$ "peak", $n p$ S "to raise, offer (in sacrificial language)" [DUL 634], Hbr. $n w p$ II: nōp "Erhebung, Höhe", *nāpāh, cstr. nāpat-, pl. nāpōt "Erhebung, Höhenzug" [GB 494, 515] = nōp "height, towering", *nāpāh, cstr. nāpat-, pl. nāpōt "yoke" [KB 683, 708], MHbr. nwp "(eig.) sich erheben", hifil "erheben, in die Höhe schwingen", npp piel "in die Höhe treiben, hoch wachsen lassen" [Levy 1924 III, 361] | OSA (Sabaean) $n w f$ "to enrich (?)" [Biella 1982, 298], Ar. $n w f$ I "1. être long et haut (un chameau), 2. être saillant, et surplomber au-dessus de qqch.", nawf- "1. sommet de la bosse d'un chameau", nyf: nāfa II "1. être augmenté, s'accroître, 2. dépasser un nombre, un chiffre", IV "dépasser, excéder" [BK II 1369, 1376] = nwf I \& IV "überragen", nawf- nüf- "Kamelhöcker" [GB] = nwf "to be high, elevated", nawf- "tip of the hump of a camel" [KB] = nawf- "Überschuß" [WUS \#1813], also Ar. $n f y / w$ : nafä "2. emporter, enlever (torrent ou vent qui enlève tout sur son passage)" [BK II 1317], MLAr. năfa "hoch und erhaben sein, übertreffen, übersteigen, darüber hinausgehen", nawf- "Plus, Überguß" [WeHR 1330], Yemeni Ar. mi-nyāf "tall stature", nāyif-ah, pl. nawāyif "lofty mountaines" [PiAmENTA 1990, 501] || Mhr. nauf "Spitze (des Pfeils)", mináuf "Gipfel" [Hein apud Müller] (Sem.: MüLler 1985, 272; DL 1990, 67-74) || Eg. $n f n f$ "das Überschwemmungswasser" (GR, Wb II 252, 8-9) = "flood water" (PL 515) = "écoulements" (Aufrère 1990, 611) || NBrb.: Shilh a-nfa "colline, sommet" [JoRdAN 1934, 35] || SBrb.: Ayr a-nəf "monter sur, s'accrocher à" [PAM 2003, 592] ||| Bed. nif "benign tumour or swelling" [Hds. 1996 MS, 99] || NOm.: Kaffa naf "gonfiarsi, esser gonfio" [Crl. 1951, 478] ||| WCh.: Goeamay niaap "to add, increase" [Srl. 1937, 159; TAKÁCs 2004, 273: 
isolated in Angas-Sura] || ECh.: WDangla nyàpè "monter sur un animal” [FÉDRY 1971, 253], EDangla nyípé "monter (sur qqch. qui avance: animal ou véhicule)" [DM 1973, 223].

884. AA * $\boldsymbol{n}-\boldsymbol{f}-\boldsymbol{t}$ "to loosen" > Sem.: Yemeni Ar. $n$ afat "to loosen, untie" [PiAmenta 1990, 491] || Eg. nft "verziehen, verdrehen" (Med., Wb II 263, 3) = "to slacken (bow), detatch, loosen" (FD 132) = "lösen, lockern (auch Bogensehne)" (GHWb 411).

885. AA * $\boldsymbol{n}-\boldsymbol{f}-\boldsymbol{T}$ "to grow" > Sem.: Ar. nafata "1. bouillonner au point de faire jaillir au dehors les gouttes du contenu (un pot), 3. être arrosé de lait ou d'eau au point de se gonfler (p.ex., farine ou autre chose en poudre)" [BK II 1303] ||| Eg. *nfd (?) > Cpt.: (SB) NOY4T, (S) NOYBT "schwellen, ausdehnen", (S) N(€)чT€ (pl.) "Schwellung, Blähung" (KHW

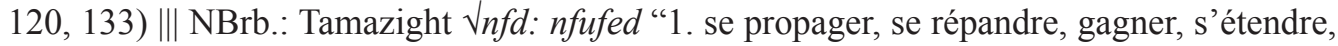
2. envahir (en se propageant), 3. se couvrir de petits boutons, s'infecter" [TAIFI 1991, 470] | Qabyle nfufed "gagner, se propager (feu)" [Dlt. 1982, 549] ||| CCh.: Fali-Jilbu nàft \& FaliMuchella naft "to fill" [KRAFT 1972 MS].

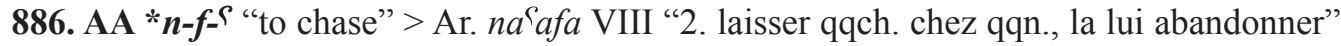
[BK II 1295] || Jbl. na $a^{\Upsilon a} f$ "to chase" [Jns. 1981, 178] || Eg. $n f^{\Upsilon}$ "1. (das Gewölk) vertreiben (PT), 2. (MK) sich eilig davon machen, 3. (GR) (die Herzen der Bösen) ausreißen” (Wb II 252, 4-6) = "loslösen, entfernen, vertreiben, herausschneiden" (KEES 1922, 112, §28)= "to push back" (PT, Allen 1984, 558) ||| (?) Bed. nif ${ }^{\Upsilon}$ "to dust, brush, sweep" (GT: lit. *"to expel dust"?) [Rpr. 1928, 222]. Diverse unconvincing etymologies of Eg. $n f^{\Upsilon}$ have so far been published, see Vcl. 1933, 180, \#10; Lsl. 1962, 47, \#28; Rsl. 1971, 288; OS 1992, 197; HSED \#1894.

887. AA * $\boldsymbol{n}-\boldsymbol{f}-\boldsymbol{r}$ "to blow the nose" > Sem.: JNAram. $n p \boldsymbol{r}$ "to blow the nose" [SABAR 2002, 234] || Eg. nf3 [reg. < *nfr] "ausniesen, (aus)schnauben" (CT, Wb II 252, 3; Osing 1986, 209, n. a) = "respirer, souffler" (LACAU 1972, 36, §43, \#5) = "expirer, expulser (du nez)" (CANnuyer 1983, 26) = "to blow, exhale" (DCT 222) ||| NBrb. *n-f-r "to exhale" [GT]: Nefusa e-nfer "se moucher" [Mtl. 1904, 138] | Tamazight (Zemmur) nfer "se moucher", $a$-nfur, pl. a-nfur-n "1. (gros) nez, 2. narine" [TAIFI 1991, 473] | Qabyle neffer "1. exhaler, expirer, souffler (l'air, fumée, vapeur), 2. fumer” [Dlt. 1982, 551] || SBrb.: Ahaggar e-nfer [Fcd. 1951-1952, 1319-1321], ETawllemmet \& Ayr o-nfor "renâcler (cheval, âne, chèvre, personne)", nəfərnəfar "1. ronfler longuement par le nez (personne, animal), renâcler, s'ébrouer, frémir, expulser l'air avec bruit, 2. inspirer l'air avec bruit par le nez" [PAM 2003, 595-596]. In the literature (GÄSW 34, \#57; CONTI 1978, 96; EHret 1995, 316, \#611; FRANCI 2005, 60), Eg. $n f 3$ has been so far directly derived from AA *n-f "to blow", while the authors were all overlooking its direct triconsonantal cognates suggesting $* n-f-r$ for the PAA level (cf. EDE I 126-127).

888. AA *n-f-r "to flee" > Sem. *npr: Ug. npr (PN) [GoRdon 1955, 298, \#1268; 1965, 446,

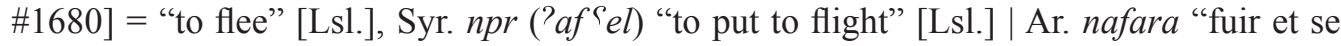
disperser, 3. s'enfuir, se sauver (gazelle), 5. s'élancer le premier pour faire qqch., 7. mettre qqn. en fuite, en déroute" [BK II 1307-1308] = “jaillir (eau), s'épouvanter (en parlant d'un troupeau), fuir çà et là etc.” [Dozy II 698, 700], Dathina nafar “s'éloigner, s'enfuir, se réfugier" [GD 2806] = "to jump" [Lsl.] || MSA: Jbl. nfor "to run down the mountain" [Jns. 1981, 182], Mhr. nəfür "to run down the mountain, usually in a panic" [Jns. 1987, 284], Sqt. $n f r$ : 
Pénfer "délier, racheter, sauver" [Ls1. 1938, 271] | Geez nafara "to fly" [Lsl.], Tigre näfra "to jump" [Lsl.], Tna. näfärä "to fly away", näfrärä "to run away" [Lsl.] (Sem.: Lsl. 1938, 271; 1987, 389) ||| Eg. nf3f3 [reg. < *nfrfr] "sauter (comme un puce)" (CT I 216a, AL 78.2088) = "to creep" (AECT I 45; DCT 222).

889. AA * $\boldsymbol{n}-\boldsymbol{f}-\boldsymbol{r}$ "young man" > Sem.: (?) Ar. nafar- "Mannschaft, insbesonders kriegerische" [Brk.] ||| Eg. nfr.w "Jünglinge (bes. die Rekruten), Jungmannschaft" (MK, Wb II 258; KAPLONy 1984, 523f.) = "Kadett" (II.?/III., Fischer, JNES 18, 1959, 258-259; OMRO 41, 1960, 7-13; FÄW 238-239) = "Jüngling, Kadett" (IV., Pusch 1974, 21) = "Rekruten (nicht Elitetruppen)" (Helck, LÄ IV 133, n. 22) = "recruits” (MK, WARD 1982, 99, \#829) = “adolescents" (Vcl. 1991, 122) = "young men (recruits, cadets, conscripts)" (Jones 2000, 453, \#1697) ||| NAgaw: Bilin onfa > omfa "child, boy" [Apl.], Hamir affa ffa afora "child, boy" [Apl.], Hamtanga amfära "servant, boy" [Apl.], Kemant anfära -m- "child, boy" [Apl.], Kailinya z-fara "servant, boy" [Faitlovitch apud Apl.], Qwara ənfära "servant, boy" [Rn.], Falasha imfera "servant, boy" [Flad apud Apl.] (NAgaw: Apl. 1984, 39; 1996, 17). For Ar.-Eg. see Brk. 1932, 106, \#32. For Eg.-NAgaw see CAStellino 1984, 16.

890. AA * $\boldsymbol{n}-\boldsymbol{f}-\boldsymbol{y}$,,to chase” > Sem. *npy: Ug. npy ,,verstossen” [WUS \#1816] | Ar. nfy I: nafā "chasser, expulser, bannir, nier, refuser qqch." [BK II 1317] = "vertreiben, verstossen" [WUS], Dathina $n f y$ "expulser, chasser" [GD 2810] || Jbl. nfy: refl. ntúfi "sich entfernen" [Bittner 1917, 54] ||| NBrb.: Izdeg $n f u$ "proscrire (homme)" [Mrc. 1937, 206] | Zwawa e-nfu “exiler, expulser" [Blf. 1910, 214].

891. AA *n-P "to open" > Sem.: Ar. nafnaf- "1. précipice entre deux rochers, 2. tout l'espace compris entre le terre et la ciel" [BK II 1317] ||| NBrb.: Tamazight $a$-nef "1. ouvrir, être ouvert, 3. s'écarter, être écarté" [TAIFI 1991, 469].

892. AA *n-P-؟ "to be fertile, useful" > Sem.: Ug. $n p$ 个 "to flourish" [GoRdon 1955, 298, \#1265] | Ar. nafa a "être utile, avantageux à qqn., servir à qqch., profiter, être bon pour la santé" [BK II 1314] = "to be useful, become of value, strong" [Lsl. 1987, 387] | MSA *nf c: Jbl. nífa؟ "1. to be useful, of use, 2. to cure" [Jns. 1981, 181] ||| ECh.: EDangla nàpàanē "être utile, rendre service", nápàanē "utile, utilisable, commode, convenable, usuel, valable, avantageux, profitable, fructueux, précieux, fertile, fécond, propice, favorable, efficace, salutaire", nàpkìye "être utile, rendre service, fructifier, être nécessaire" [DM 1973, 214], cf. also WDangla nàpkìyè "profiter de son labeur" [FÉDRY 1971, 214] | Mokilko 'ámpè "valoir, être utile, utilisable, convenable" [Jng. 1990, 58].

893. AA *n-P-l "loose skin (???)" > Sem.: Hbr. npl: *mappāl in mappalē-bəŝārōo "seine fleischigen Wampen" [GB 449] = mappolē-bā̂̀̄ar "folds of flesh" [KB 618] ||| EBrb.: Ghadames $a$-naful, pl. nüfal "outre de peau pour conserver des provisions sèches: farine, etc. ..., sauf les dattes", ta-naful-t, pl. t-nufal "petite outre" [LANFrY 1973, 237, \#1110].

\section{ABBREVIATIONS OF LANGUAGES}

(A): Akhmimic, AA: Afro-Asiatic, Akk.: Akkadian, Ar.: Arabic, Aram.: Aramaic, AS: Angas-Sura, (B): Bohairic, Bed.: Bed'awye, Brb.: Berber, BT: Bole-Tangale, Ch.: Chadic, CCh.: Central Chadic, CT: coffin texts, Cu.: Cushitic, ECh.: East Chadic, ECu.: East Cushitic, Dhl.: Dahalo, E: East(ern), Eg.: Egyptian, EWlm.: East Tawllemmet, (F): Fayyumic, GR: Greek (Ptolemaic) and Roman Period, GW: syllabic or group-writing, HECu.: Highland 
East Cushitic, Hgr.: Ahaggar, IMP: Intermediate Period, Irq.: Iraqw, JAram.: Jewish Aramaic, (L): Lycopolitan (or Subakhmimic), LECu.: Lowland East Cushitic, Lit.: literary texts, LP: Late Period, M: Middle, MK: Middle Kingdom, N: North, NBch.: North Bauchi, NBrb.: North Berber, NK: New Kingdom, NOm.: North Omotic, OEg.: Old Egyptian, OK: Old Kingdom, Om.: Omotic, PCh.: Proto-Chadic, PCu.: Proto-Cushitic, PT: pyramid texts, Qbl.: Qabyle, Qwd.: Qwadza, S: South(ern), (S): Sahidic, Samar.: Samaritan, SBrb.: South Berber, Sem.: Semitic, W: West(ern), WBrb.: West Berber, WCh.: West Chadic, WSem.: West Semitic.

\section{ABBREVIATIONS OF AUTHOR NAMES}

AJ: Alio \& Jungraithmayr, Apl.: Appleyard, BK: Biberstein Kazimirsky, Blf.: Boulifa, Bnd.: Bender, Brk.: Brockelmann, Brt.: Barreteau, Bst.: Basset, CR: Conti Rossini, Crl.: Cerulli, Dl: Dietrich \& Loretz, Dlt.: Dallet, DM: Duibrine \& Montgolfier, Ebs.: Ebobisse, Fcd.: Foucauld, Fdr.: Fédry, Ftp.: Fitzpatrick, GB: Gesenius \& Buhl, GT: TakÁcs, Hds.: Hudson, Hlw.: Hellwig, Hnrg.: Huehnergard, Hyw.: Hayward, JA: Jungraithmayr \& Adams, Jng.: Jungraithmayr, Jns.: Johnstone, KB: Koeehler \& Baumgartner, Krf.: Kraft, Lbg.: Loubignac, LH: LitTMann \& Höfner, Lnf.: Lanfry, Lsl.: Leslau, LSS: Lonnet \& Simeone-Senelle, Lst.: Laoust, Mrc.: Mercier, Mtl.: Motylinsky, Nhl.: Nehlit, OS: Orel \& Stolbova, PAM: Prasse \& Alojaly \& Mohamed, PG: Pillinger \& Galboran, PH: Parker \& Hayward, Prs.: Prasse, Rdk.: Rhodokanakis, Rn.: Reinisch, Rns.: Renisio, Rpr.: Roper, Rsl.: Rössler, SS: Simeone-Senelde, Srl.: Sirlinger, Stl.: Stolbova, Vcl.: Vycichl.

\section{LITERATURE}

AECT $=$ FAULKNER Raymond. 1973-1978. The Ancient Egyptian Coffin Texts. Vol. I-III. Warminster: Aris \& Phillips Ltd.

AHW $=$ SoDEN Wolfram von. 1965-1981. Akkadisches Handwörterbuch. I-III. Wiesbaden: Otto Harrassowitz.

AL I-III = Meeks Dimitri. 1998. Année lexicographique. Égypte ancienne. Tome 1-3 (1977-1979). $2^{2 \mathrm{eme}}$ édition. Paris: Cybele.

Alio Khalil, Jungratthmayr Herrmann. 1989. Lexique bidiya. Frankfurt am Main: Vittorio Klostermann.

Allen Thomas George. 1984. The Inflection of the Verb in the Pyramid Texts. Bibliotheca Aegyptia, vol. 2, fasc. 1-2. Malibu: Undena Publications.

Altheim F., Stienl R. (eds.). 1971. Christentum am Roten Meer. Band I. Berlin-New York: Walter de Gruyter.

Appleyard David. 1984. "The Internal Classification of the Agaw Languages. A Comparative and Historical Phonology.” In: BYNON 1984: 33-67.

Appleyard David. 1996. “'Kaïliña' - A 'New' Agaw Dialect and Its Implications for Agaw Dialectology.” In: HaYwARD \& LEWIS 1996: 1-19.

AтזYA A.S. (ed.). 1991. The Coptic Encyclopaedia. Vol. 8. New York: MacMillan.

Aufrère Sydney. 1990-1991. L'univers minéral dans la pensée égyptienne. Vol. I-II. BdÉ 105. Le Caire: IFAO.

Barreteau Daniel, Bléis Yvonne. 1990. Lexique mafa. Langue de la famille tchadique parlée au Cameroun.

Paris: ORSTOM, Librairie Orientaliste Paul Geuthner.

Barreteau Daniel, Brunet André. 2000. Dictionnaire Mada. Berlin: Dietrich Reimer Verlag.

BASSET René. 1890. Loqmân berbère avec quatre glossaires et une étude sur la légende de Loqmân. Paris: Ernest Leroux.

BASSET René. 1890. Le dialecte de Syouah. Paris: Ernest Leroux.

Bender Marvin Lionel. 1971. "The Languages of Ethiopia. A New Lexicostatistic Classification and Some Problems of Diffusion." Anthropological Linguistics 13(5), 165-288.

BENDER Marvin Lionel. 1994. "Aroid (South Omotic) Lexicon." Afrikanistische Arbeitspapiere 38, 133-162.

Bender Marvin Lionel. 1994. “The Mystery Languages of Ethiopia.” In: Marcus 1994: 1153-1174.

Bender Marvin Lionel. 1996. Wordlists of Dimé, Dizi, Nayi, Sheko. MS. 5 August 1996.

Bender Marvin Lionel. 2003. Omotic Lexicon and Phonology. Carbondale: SIU Printing/Duplicating, Southern Illinois University.

BibersteIn Kazimirski Albert de. 1860. Dictionnaire arabe-français. Paris: Maisonneuve \& Co. Editeurs.

Biella Joan Copeland. 1982. Dictionary of Old South Arabic. Chico: Scholars Press at Harward.

BitTNER Maximilian. 1917. "Studien zur Šhauri-Sprache in den Bergen von D́àofâr am Persischen Meerbusen.

IV. Index (šhauri-deutsches Glossar) und Nachträge zu den Texten von D.H. von Müller (textkritische Noten 
nach den ersten Aufnahmen)." Sitzungsberichte der Kaiserlichen Akademie der Wissenschaften in Wien, phil.-hist. Klasse, 183. Band, 5. Abhandlung, 1-106.

Boulifa Saïd A. 1910. Une première année de langue kabyle (dialecte zouaoua) à l'usage des candidats à la prime et au brevet de kabyle. Deuxième édition. Alger: Adolphe Jourdan.

BRockelmann Carl. 1932. “Ägyptisch-semitische Etymologien.” Zeitschrift für Semitistik 8, 97-117.

Bynon J. (ed.). 1984. Current Progress in Afro-Asiatic Linguistics. Amsterdam. Philadelphia: John Benjamins.

Bynon J., Bynon Th. (eds.). 1975. Hamito-Semitica. The Hague: Mouton de Gruyter.

$\mathrm{CAD}=$ The Assyrian Dictionary of the Oriental Institute of the University of Chicago. Vol. 1-21. GlückstadtChicago, Since 1956. J.J. Augustin, The Oriental Institute of the University of Chicago.

CANNUYER Christian. 1983. "Les formes dérivées du verbe en ancien égyptien. Essai de systématisation." GM 63, $25-33$.

CAstellino Giorgio Raffaele. 1984. "Relazione introduttiva." In: Atti della Terza Giornata di Studi Camito-Semitici e Indoeuropei. Roma: Università degli Studi "La Sapienzia", 8-18.

CED $=$ ČERnÝ Jaroslav. 1976. Coptic Etymological Dictionary. London-Cambridge: Cambridge University Press.

CERULli Enrico. 1951. Studi etiopici. IV. La lingua caffina. Roma: Istituto per l'Oriente.

ConTI Giovanni. 1978. Rapporti tra egiziano e semitico nel lessico egiziano dell'agricoltura. Firenze: Istituto di Linguistica e di Lingue Orientali, Università di Firenze.

Conti Rossini Carlo. 1913. Schizzo del dialetto saho dell'alta Assaorta in Eritrea. Roma: Tipografia della R. Accademia dei Lincei.

Conti Rossini Carlo. 1913. "Studi su populazioni dell'Etiopia." Rivista degli Studi Orientali 6, 365-426.

CT = BucK Adrian de. 1935-1961. The Egyptian Coffin Texts. Vol. I-VII. Chicago: The University of Chicago Press.

DAноOD Mitchell. 1976. "Hebrew Lexicography: A Review of W. Baumgartner's Lexikon.” Vol. II. Orientalia NS 45, 327-365.

Dallet J.-M. 1982. Dictionnaire qabyle-français. Parler des At Mangellat (Algerie). Paris: SELAF (Société d'études linguistiques et anthropologiques de France).

Dalman Gustaf H. 1922. Aramäisch-neuhebräisches Handwörterbuch zu Targum, Talmud und Midrasch. Frankfurt a/M: J. Kaufmann Verlag.

DCT $=$ Molen Rami van der. 2000. A Hieroglyphic Dictionary of Egyptian Coffin Texts. Leiden: E.J. Brill.

DELC = Vycichl Werner. 1983. Dictionnaire étymologique de la langue copte. Leuven: Peeters.

Dietrich M., Loretz O. 1990. "Die Wurzel nūp “hoch sein' im Ugaritischen.” UF 22, 67-74.

Disirine Bada Adoum Zaid, Montgolfier Paul de (etc.). 1973. Vocabulaire dangaléat. Kawo dayla. Place not indicated, around. (deduced by G. Takács), publisher not indicated.

DNWSI = HoftiJzer Jacob, Jongeling K. 1995. Dictionary of North-West Semitic Inscriptions. Part 1-2. Leiden: E. J. Brill.

Dozy R. 1881. Suppléments aux dictionnaires arabes. Tome I-II. Leiden-Paris: E. J. Brill, Maisonneuve.

DUL $=$ Olmo Lete Gregorio, SAnMartín Joaqín. 2003. A Dictionary of the Ugaritic Language in the Alphabetic Tradition. Part One [?(a/i/u)-k]. Part Two [l-z]. Leiden: E.J. Brill.

EbBell Bendix. 1937. "Ägyptische anatomische Namen.” Acta Orientalia 15, 293-310.

EвoBISSE Carl. 1979. Die Morphologie des Verbs im Ost-Dangaleat (Guera, Tschad). Berlin: Dietrich Reimer Verlag.

EвоBisse Carl. 1987. Les verbaux du dangaléat de l'est (Guera, Tchad). Lexiques français-dangaléat et allemanddangaléat. Berlin: Dietrich Reimer Verlag.

EDE I = TAKÁCS Gábor. 1999. Etymological Dictionary of Egyptian. Volume One: A Phonological Introduction. Leiden: E. J. Brill.

EHRET Christopher. 1995. Reconstructing Proto-Afroasiatic (Proto-Afrasian). Vowels, Tone, Consonants, and Vocabulary. Berkeley, Los Angeles, California: University of California.

Ember Aaron. 1917. “Kindred Semito-Egyptian Words (New Series)." Continued from Vol. 51, pp. 110-121. ZÄS 53, 83-90.

Fauvelle-Aymar F.-X., Chrétien J.-P., Perrot C.-H. (eds.). 2000. Afrocentrismes. L'histoire des Africains entre Égypte et Amérique. Paris: Éditions Karthala.

FÄW(b) = KaHL Jochem. 2002-2004. Frühägyptisches Wörterbuch (unter Mitarbeit von M. Bretschneider und B. Kneißler). Wiesbaden, Lieferung 1-4. Wiesbaden: Harrassowitz.

FD $=$ FAULKNER Raymond O. 1962. A Concise Dictionary of Middle Egyptian. Oxford: Clarendon Press. 
Fеснт Gerhard. 1960. Wortakzent und Silbenstruktur. Untersuchungen zur Geschichte der ägyptischen Sprache. Glückstadt: Verlag J. J. Augustin.

FÉDRY Jacques (avec la collaboration de Khamis, J. \& o/Nedjei, M.). 1971. Dictionnaire dangaleat (Tchad). Thése de $3^{\text {ème }}$ cycle, Institut National des Langues et Civilisations Orientales. Lyon: Afrique et Langage.

FitzPatrick J.F.J. 1910-1911. "Some Notes on the Kwolla District and Its Tribes." Journal of the Royal African Society 10, 16-52, 213-222.

Foucauld Charles de. 1951-1952. Dictionnaire touareg-français, dialecte de l'Ahaggar. Vol. I-IV. Paris: Imprimerie Nationale de France.

Franci Massimiliano. 2005. "Egypto-Semitic Lexical Comparison: New Correspondences and Phonological Problems in the Lexicon of Anatomy and Physiological Functions." In: Mengozzi 2005: 57-66.

GÄSW = CALICE Franz von. 1936. Grundlagen der ägyptisch-semitischen Wortvergleichung. Wien: Selbstverlag des Orientalischen Institutes der Universität Wien.

$\mathrm{GB}=$ GesENIUS Wilhelm (bearbeitet von Buhl, Frants). 1962. Hebräisches und aramäisches Handwörterbuch über das Alte Testment. Unveränderter Neudruck der 1915 erschienenen 17. Auflage. Berlin-GöttingenHeidelberg: Springer-Verlag.

GD = LandBerg Le Comte de. Glossaire datînois. Vol. I (1920), II (1923), III (1942). Leiden: E. J. Brill.

$\mathrm{GHWb}=$ HaNNIG Rainer. 1995. Grosses Handwörterbuch Ägyptisch-Deutsch (2800-950 v. Chr.). Mainz: Verlag Philipp von Zabern.

Gordon Cyrus H. 1955. Ugaritic Manual. Roma: Pontificium Institutum Biblicum.

Gordon Cyrus H. 1965. Ugaritic Textbook. Roma: Pontificium Institutum Biblicum.

Grapow Hermann. 1954. Anatomie und Physiologie (Grundriß der Medizin der Alten Ägypter I.). Berlin: Akademi-Verlag.

Guillaume Alfred. 1965. Hebrew and Arabic Lexicography. A Comparative Study. Reprinted from: Abd-Nahraim. Vol. I-IV (1959-1965). Leiden: Brill.

HARris Zellig S. 1936. A Grammar of the Phoenician Language. New Haven, Connecticut: American Oriental Society.

HAYWARD Richard J. 1984. The Arbore Language: A First Investigation Including a Vocabulary. Hamburg: Helmut Buske Verlag.

HAYWARD Richard J. 1989. "Comparative Notes on the Language of the S'amakko." JAAL 1, 1-53.

HaYward Richard J., Lewis I.M. (eds.). 1996. Voice and Power. The Culture of Language in North-East Africa. London: SOAS.

Hegedús I., Sidwell P. (eds.). 2004. Nostratic Centennial Conference: The Pécs Papers. Pécs: Lingua Franca Group.

Hellwig Birgit. 2000. Goemai - English - Hausa Dictionary. MS. Draft. Printed out on 20 August 2000. 42 p.

Hetzron R. (ed.). 1997. The Semitic Languages. London-New York: Routledge.

Hornung Erik. 1963. Das Amduat. Die Schrift des verborgenen Raumes nach Texten aus den Gräbern des Neuen Reiches. Teil I: Text. Teil II: Übersetzung und Kommentar. Teil III: Die Kurzgefassung. Wiesbaden: Otto Harrassowitz.

HSED = Orel Vladimir É., Stolbova Ol'ga V. 1995. Hamito-Semitic Etymological Dictionary. Leiden: E.J. Brill.

Hudson G. 1989. Highland East Cushitic Dictionary. Hamburg: Buske.

Hudson Richard A. 1996. A Dictionary of Beja. Draft Printout. February 29, 1996. Version prepared by R.M. Blench.

HueHnergard John. 1987. Ugaritic Vocabulary in Syllabic Transcription. Harvard Semitic Studies 32. Atlanta: Scholars Press.

HueHneRgard John. 1999. "On the Etymology and Meaning of Hebrew nābî'." Eretz-Israel 26, 88-93.

JACOBSON Howard. 2001. "Nebel." Zeitschrift für Althebraistik 14, 84-85.

JASTROw Marcus. 1950. A Dictionary of the Targumim, the Talmud Babli and Yerushalmi, and the Midrashic Literature. Vol. I: '-k, Vol. II: 1-t. New York: Pardes Publishing House Inc.

JNES = Journal of Near Eastern Studies (Chicago).

Johnstone T.M. 1977. Harsūsi Lexicon. London: Oxford University Press.

Johnstone T.M. 1981. Jibbāli Lexicon. London: Oxford University Press.

Johnstone T.M. 1987. Mehri Lexicon. London: University of London.

Jones Dilwyn. 2000. An Index of Ancient Egyptian Titles, Epithets and Phrases of the Old Kingdom. Vol. I. Oxford: Archaeopress, Publishers of the British Archaeological Reports. 
JORDAN Antoine. 1934. Dictionnaire berbère-français (dialectes tašelhait) contenant 6.025 formes. Rabat: Éditions Omnia.

Junge F. (Hrsg.). 1984. Studien zur Sprache und Religion Ägyptens. Band 1: Sprache. Zu Ehren von Wolfhart Westendorf überreicht von seinen Freunden und Schülern. Göttingen: Hubert \& Co.

Jungratthmayr Hermann. 1990. Lexique mokilko. Berlin: Dietrich Reimer Verlag.

JungratthmaYr Hermann, Adams Abakar. Lexique migama. Berlin: Dietrich Reimer Verlag.

JuŠmAnov N.V. 1998. Izbrannye trudy. Raboty po obščej fonetike, semitologii i arabskoj klassičeskoj morfologii. Ed. by Anna G. Belova. Moskva: Izdatel'skaja firma "Vostočnaja literatura" RAN.

KaPlony Peter. 1984. "Ein 'Senior der Jungmannschaft' der Frühzeit und seine Opferliste (SP 53).” In: Junge 1984: 521-546.

KB $=$ KoenLer Ludwig, Baumgartner Walter. 1994-2000. The Hebrew and Aramaic Lexicon of the Old Testament. I-V. Leiden: E. J. Brill.

KeEs Hermann. 1922. "Ein alter Götterhymnus als Begleittext zur Opfertafel.” Z̈̈S 57, 92-120.

KHW = WESTENDORF Wolfhart. 1977. Koptisches Handwörterbuch. Heidelberg: Carl Winter Universitätsverlag.

Kraft Charles H. 1972. Gude-Dialekte. MS.

LACAu Pierre. 1972. Études d'Égyptologie. II. Morphologie. Le Caire: IFAO.

Ladefoged Peter. 1964. A Phonetic Study of West African Languages. An Auditory-Instrumental Survey. Cambridge: University Press.

Lane Edward William. 1863-1893. An Arabic-English Lexicon. I-VIII. London-Edinburgh: Williams and Norgate.

LANFRY J. 1973. Ghadames. II. Glossaire. Alger: Le Fichier Periodique.

Laoust Émile. 1921. Cours de berbère marocain. Grammaire - vocabulaire - textes. Dialectes du sous du Haut et de l'Antiatlas. Paris: Augustin Challamel.

LÄ = HeLck Wolfgang, WestendorF Wolfhart (Hrsg., begründet von W. Helck und E. Otto). 1975-1992. Lexikon der Ägyptologie. Band I-VII. Wiesbaden: Harrassowitz.

LESLAU Wolf. 1938. Lexique soqotri (sudarabique moderne), avec comparaisons et explications étymologiques. Paris: Librairie C. Klincksieck.

Leslau Wolf. 1956. "Additional Notes on Kambatta of Southern Ethiopia." Anthropos 51, 985-993.

Leslau Wolf. 1958. Ethiopic and South Arabic Contributions to the Hebrew Lexicon. Berkeley-Los Angeles: University of California.

Leslau Wolf. 1962. "Semitic and Egyptian Comparisons." Journal of Near Eastern Studies 21, 44-49.

Leslau Wolf. 1962. "A Prefix in Egyptian, Modern South Arabian, and Hausa." Africa 32, 65-68.

LesLau Wolf. 1987. Comparative Dictionary of Ge'ez (Classical Ethiopic). Wiesbaden: Otto Harrassowitz.

LEVY Jacob. 1924. Wörterbuch über die Talmudim und Midraschim nebst Beiträgen von Heinrich Leberecht Fleischer. Zweite Auflage mit Nachträgen und Berichtigungen von Lazarus Goldschmidt. I-IV. BerlinWien: Benjamin Harz Verlag.

LitTMann Enno, Höfner Maria. 1956. Wörterbuch der Tigre-Sprache. Tigre-Deutsch-Englisch. Wiesbaden: Franz Steiner Verlag.

Lonnet Antoine, Simeone-Senelle Marie-Claude. 1983. "Observations phonétiques et phonologiques sur les consonnes d'un dialecte mehri." Matériaux Arabes et Sudarabiques 1, 187-218.

LonNET Antoine. 1993. "Quelques résultats en linguistique sudarabique moderne.” Quaderni di Studi Arabi 11, 37-82.

LORET Victor. 1896. "Les animaux reproducteurs dans l’Égypte ancienne.” RT 18, 196-209.

Loubignac V. 1924. Étude sur le dialecte berbère des Zaïan et Aït Sgougou. Textes (deuxième section). Paris: Ernest Leroux.

Marcus H. (ed.). 1994. New Trends in Ethiopian Studies. Vol. 1. Lawrenceville: Red Sea Press.

Mathieu Bernard. 1996. La poésie amoureuse de l'Égypte ancienne. Recherches sur un genre littéraire au Nouvel Empire. Le Caire: IFAO.

Mengozzi A. (ed.). 2005. Studi afroasiatici. XI Incontro Italiano di Linguistica Camitosemitica. Afro-Asiatic Studies: $11^{\text {th }}$ Italian Meeting of Afro-Asiatic Linguistics. Milano: Francoangelli.

MerCIER Henry. 1937. Vocabulaires et textes berbères dans le dialecte des Ait Izdeg. Rabat: René Céré.

MotyLinski A. de C. 1904. Le dialecte berbère de R'edamès. Paris: Ernest Leroux.

MüLLER Walter W. 1975. "Beiträge zur hamito-semitischen Wortvergleichung.” In: BYNON \& BYNON 1975: 6374. 
MülLER Walter W. 1985. "Beiträge aus dem Mehri zum etymologischen Teil des hebräischen Lexikons.” In: Mélanges linguistiques offerts à Maxime Rodinson. Paris: Librairie Orientaliste Paul Geuthner, 267-278.

NBÄ = Osing Jürgen. 1976. Die Nominalbildung des Ägyptischen. I-II. Maiz/Rhein: Verlag Philipp von Zabern. NeHLIL. 1909. Étude sur le dialecte de Ghat. Paris: Éditions Ernest Leroux.

OMRO = Oudheidkundige Mededelingen uit het Rijksmuseum van Oudheden te Leiden (Leiden).

Or. $=$ Orientalia $($ Roma) .

Orel Vladimir É., Stolbova Ol'ga V. 1992. “Cushitic, Chadic, and Egyptian: Lexical Relations.” In: ShevoroshKIN 1992: 167-180.

Orel Vladimir É., Stolbova Ol'ga V. 1992. “On Chadic-Egyptian Lexical Relations.” In: Shevoroshkin 1992: 181-203.

Osing Jürgen. 1980. "Besprechung von Ward, W.A. The Four Egyptian Homographic Roots B-3." Bibliotheca Orientalis 37(3-4), 139-140.

Osing Jürgen. 1986. "Sprüche gegen die jbh3tj-Schlange." Mitteilung des Deutschen Archäologischen Instituts Abteilung Kairo 43, 205-210.

Parker Enid M., Hayward Richard J. 1985. An Afar-English-French Dictionary (with Grammatical Notes in English). London: School of Oriental and African Studies, University of London.

Piamenta Moshe. 1990-1991. Dictionary of Post-Classical Yemeni Arabic. I-II. Leiden: Brill.

Pillinger Steve, Galboran Letiwa. 1999. A Rendille Dictionary. Köln: Rüdiger Köppe Verlag.

PL $=$ WILSON Penelope. 1997. A Ptolemaic Lexikon. A Lexicographical Study of the Texts in the Temple of Edfu. Leuven: Peeters.

PRASSE Karl-G. 1969. "A propos de l'origine de h touareg (tahaggart)." Det Kongelige Danske Videnskabernes Selskab. Historisk-filosofiske Meddelelser 43(3).

Prasse Karl-G., Alojaly Gh., Mohamed Gh. 2003. Dictionnaire touareg-français (Niger). Copenhagen: Museum Tusculanum Press, University of Copenhagen.

PT $=$ SeTHE Kurt. 1980 (1910). Die altägyptischen Pyramidentexte. I-II. Leipzig: J.C. Hinrichs'sche Buchhandlung.

Pusch Edgar. 1974. "Register der ägyptischen Wörter zu Junker Giza I-XII.” Z̈̈S 101, 13-35.

ReInIsch Leo. 1886. "Die 'Afar-Sprache. II." Sitzungsberichte der Kaiserlichen Akademie der Wissenschaften. Phil.-hist. Classe 113(2), 795-916.

ReInISCH Leo. 1887. Wörterbuch der Bilin-Sprache. Wien: Alfred Hoelder.

ReINISCH Leo. 1888. "Die Kafa-Sprache in Nordost-Afrika. II. Kafa-Deutsches Wörterbuch.” Sitzungsberichte der Kaiserlichen Akademie der Wissenschaften. Phil.-hist. Classe 116, 251-386.

ReINISCH Leo. 1890. Wörterbuch der Saho-Sprache. Wien: Alfred Hölder.

ReNisio A. 1932. Étude sur les dialectes berbères des Beni Iznassen, du Rif et des Senhaja de Sraïr. Grammaire, textes et lexique. Paris: Éditions Ernest Leroux.

RHodokanakis Nikolaus. 1911. Der vulgärarabische Dialekt im D'ofâr (Z'fâr). II. Einleitung, Glossar und Grammatik. Wien: Alfred Hölder.

Roper E.-M. 1928. Tu BedawiE. An Elementary Handbook for the Use of Sudan Government Officials. Hertford: Stephen Austin \& Sons.

RössLer Otto. 1971. "Das Ägyptische als semitische Sprache.” In: Altheim \& Stienl 1971: 263-325.

SABAR Yona. 2002. A Jewish Neo-Aramaic Dictionary. Dialects from Amidya, Dihok, Nerwa and Zakho, Northwestern Iraq. Wiesbaden: Harrassowitz.

Schlee Günther. 1978. Sprachliche Studien zum Rendille: Grammatik, Texte, Glossar. Hamburg: Helmut Buske Verlag.

Segert Stanislav. 1984. A Basic Grammar of the Ugaritic Language with Selected Texts and Glossary. BerkeleyLos Angeles: University of California Press.

Shevoroshrin V. (ed.). 1992. Nostratic, Dene-Caucasian, Austric and Amerind. Bochum: Brockmeyer.

ŠIFMAN I.Š. 1963. Finikijskij jazyk. Moskva: Izdatel'stvo Vostočnoj Literatury.

Simeone-Senelle Marie-Claude. 1997. “The Modern South Arabian Languages.” In: Hetzron 1997: 378-423.

Sirlinger Eugene. 1937. Dictionary of the Goemay Language. Jos, Nigeria: Prefecture Apostolic of Jos.

Stolbova Ol'ga V. 2005. Chadic Lexical Database. Issue I. L, N, NY, R. Kaluga: Poligrafiya.

Stumme Hans. 1899. Handbuch des Schilchischen von Tazerwalt. Leipzig: J. C. Hinrichs'sche Buchhandlung.

TAïFI Miloud. 1991. Dictionnaire tamazight-français (parlers du Maroc central). Paris: L'Harmattan-Awal.

TAKÁCs Gábor. 2004. Comparative Dictionary of the Angas-Sura Languages. Berlin: Dietrich Reimer Verlag. 
TAKÁCs Gábor. 2004. “Aegyptio-Afroasiatica XIX.” Rocznik Orientalistyczny 57(2), 47-89.

TAKÁCS Gábor. 2004. “Angas-Sura Etymologies II.” Rocznik Orientalistyczny 57(1), 55-68.

TAKÁCS Gábor. 2004. "Afrasian and Nostratic: Addenda to the Nostratic Root List of A. B. Dolgopolsky." In: Hegedüs \& Sidwell 2004: 193-227.

TAL Abraham. 2000. A Dictionary of Samaritan Aramaic. Vol. I-II. Leiden: E.J. Brill.

$\mathrm{UF}=$ Ugarit-Forschungen (Münster).

Vergari Moreno, Vergari Roberta. 2003. A Basic Saho-English-Italian Dictionary. Asmara, Eritrea (publisher not indicated).

Vernus Pascal. 2000. "Situation de l'égyptien dans les langues du monde." In: Fauvelle-Aymar et al. 2000: 169-208.

Vycıchl Werner. 1933. "Aigyptiaka. Beiträge zur vergleichenden Hamitosemitistik.” Wiener Zeitschrift für die Kunde des Morgenlandes 40, 171-180.

VyсіснL Werner. 1960. "Gedenken zur ägyptisch-semitischen Sprachverwandtschaft." Muséon 73, 173-176.

Vycichl Werner. 1991. "Etymology.” In: AтіYA 1991: 118-124.

Walker James H. 1996. "Studies in Ancient Egyptian Anatomical Terminology." Warminster: Aris \& Phillips Ltd.

Ward William A. 1978. The Four Egyptian Homographic Roots b3. Rome: Biblical Institute Press.

WARD William A. 1982. Index of Egyptian Administrative and Religious Titles of the Middle Kingdom. With Glossary of the Words and Phrases Used. Beirut: American University of Beirut.

$\mathrm{Wb}=$ ERMAn Adolf, GraPOw Hermann. 1957-1971. Wörterbuch der ägyptischen Sprache. I-V. ${ }^{2}$ Berlin: Akademie-Verlag.

WeHR Hans. 1952. Arabisches Wörterbuch für die Schriftsprache der Gegenwart. Leipzig: Otto Harrassowitz.

WeSTENDORF Wolfhart. 1966. "Beiträge aus und zu den medizinischen Texten." Z̈̈S 92, 128-154.

WUS = AisTLEITNER Josef. 1963. "Wörterbuch der ugaritischen Sprache." Berichte über die Verhandlungen der Sächsischen Akademie der Wissenschaften zu Leipzig. Phil.-hist. Klasse 106(3).

$\mathrm{ZA}=$ Zeitschrift für Assyriologie und Verwandte Gebiete (Berlin, New York).

ZABORSKI Andrzej. 1971. "Biconsonantal Verbal Roots in Semitic." Zeszyty Naukowe Uniwersytetu Jagiellonskiego, Prace Językoznawcze 5, 51-98.

Allatum die 5 mensis Junii anno 2010 